\title{
Inspirations for Optimization based on Benchmarking
}

\author{
A.S. XIE ${ }^{1, a}$ and D.X. LIU $^{1, b}$ \\ ${ }^{1}$ China Institute for SMEs, Zhejiang University of Technology, Hangzhou, China \\ a shermanxas@163.com, b daoxueliu@zjut.edu.cn
}

Keywords: Optimization Problems, Intelligent Optimization, Optimization Algorithm, Evolutionary Computation, Swarm Intelligence, Encoding Scheme, Benchmarking Philosophy.

\begin{abstract}
The modern intelligent optimization/search algorithms were broadly classified, and some brief analysis and comment were made. Then we revealed some optimization rules implied in the benchmarking philosophy, which provided good inspirations for designing of modern intelligent optimization/search algorithms.
\end{abstract}

\section{Introduction}

Many of the issues in science, engineering, and management can be transformed into optimization problems. For example, the problem of predicting the stability of the protein in Bioinformatics can be converted into the optimization problem of searching the lowest potential energy value of protein structure. The classification of tumor microarray gene expression data in Molecular Biology can be transformed into the optimization problem of solving the optimal tumor feature gene extraction and classification model. The problem of structural determination of complex networks in big data can be converted into the optimization problem of searching the optimal network structure. The real-time scheduling problem of modern intelligent industrial mobile robots can be turned into the optimization problem of the mobile path of robots. For these optimization problems, some of which can be solved by using traditional operations research methods, but the vast majority is impossible. For example, the field of photoelectric conversion. In the design phase of solar cells, to find the key factors of photoelectric conversion efficiency of solar cells with different materials, it needs to analyze and extract the parameters of the equivalent circuit model. At this point, we can use the traditional analytical method, although the actual effect is not as good as the intelligent method. In the practical application of solar cells, the photovoltaic array is a real-time source of electricity in a daily environment. Its output characteristic will vary with ambient temperature, sunshine intensity, and other factors, and it is a complex nonlinear form. Therefore, the maximum power points cannot be obtained simply by using an equivalent model. Also, it is hard to express in mathematical models. That is to say, we cannot use the traditional analytical method to get the maximum power points. Therefore, to improve the energy efficiency of photovoltaic power generation system and obtain the maximum power output, it is very important to keep tracking of the maximum power points. At this point, the traditional operations research methods are helpless, and it can only be helped by using intelligence method.

Genetic algorithm (GA) is the first of its kind in modern intelligent optimization algorithms (IOAs). Since it was put forward, many scholars have done much pioneering work. In recent years, there are tens of thousands of literature, and the original IOAs have more than a hundred by far. Basing on the principle of optimization and its overall characteristic, they can be divided into four categories. The first type is the evolutionary computation. There are several kinds of evolutionary algorithms and various improved versions. Although the specific search techniques are different, the same optimization idea is to imitate the evolutionary process of biology and generate candidate solutions by selecting, crossover and mutation. The second type is the swarm intelligence, which is mainly inspired by the behavior of social insects or animals. There are a large number of living species in the biosphere, so this kind of algorithms develops very quickly. Entering the 21st century, almost one or two new algorithms emerged every year. Swarm intelligence is a type of distributed algorithm, and the so-called swarm intelligence refers to that the non-intelligent individuals 
expressed the characteristics of intelligent behavior through the cooperation between each other. In the swarm intelligence system, the unit individual has simple intelligence and behavior, but this group of individuals can cooperate to solve the complex problems. So the group has a kind of whole intelligence. In swarm intelligence algorithms, there is no control center, and the interaction between individuals is also extremely simple. The most well-known of swarm intelligence is Particle Swarm Optimization and Ant Colony Optimization. The third type is the phenomenon algorithm, which is mainly inspired by the internal rules behind the various natural phenomena. The mechanism behind phenomenon algorithm is different from the evolutionary computation, and also is distinct from the swarm intelligence. Each random collection of the search agents is realized by moving or communication of the search space according to the physical rules. The search behavior is performed by analogical gravitation, weight, light, projection, inertial force, electromagnetic force etc. Because of the complexity of physical phenomena, the mechanisms and the rules of which are also varied, it can be predicted that this kind of IOAs will usher in a wave of a high tide of prosperity with the rapid development of artificial intelligence, data mining, knowledge discovery, mobile networks and other fields. The final type, which can't be put into the three previous categories. For example, the Simulated Annealing Algorithm, although derived from the solid annealing concept, has only a single candidate solution for each iteration. The Microcanonical Annealing Algorithm, although imitating the annealing mechanism, the state transfer in the process of annealing is in a deterministic way instead of using the Metropolis criterion. Taboo Search, Variable Neighborhood Algorithm, and Predatory Search, these three algorithms should not be categorized as the optimization algorithm. Which, in essence, should be considered as three unique search strategies. [1]

Of course, the division of four types is rough and not strict. In fact, there are some algorithms, whose own designs ideas have various features. For example, Memetic Algorithm, which included the concept of evolutionary computing, but also reflected the characteristic of swarm intelligence. Classifying the original IOAs into four categories, there is no special purpose. Just because it would be confusing to put so many original algorithms together. They were just classified by intuition, so the classification is rough. In the same way as traditional optimization algorithms (including operations research methods) based on strict mathematical logic, the IOAs are essentially the kind of search algorithms based on the hill-climbing model. The traditional optimization algorithms are the type of deterministic hill-climbing, i.e., the direction and step size are determined by the derivatives of the objective function. Which is also the limitation of them, i.e., the prerequisite for its application is that the problem can be built as an accurate mathematical model, and the objective function must be continuous and differentiable. However, the IOAs almost have no such restrictions. Although the search behavior of the single individual is non-deterministic, the search behavior at the population level has a strong tendency towards the "peak(s)". Usually, The IOAs are inspired by the laws of nature or behaviors of biological groups. Whose design idea is that the random sample points in the problem domain are regarded as moving individuals, and the individuals have some properties as fitness, speed, and position. Under the guidance of some probability rules, these individuals can get the possible optimal solution by continuous updating of speed and position. This search process has a certain degree of intelligence. Comparing with the operations research methods based on rigorous mathematical logic, although these methods have been applied satisfactorily in practice, there is no rigorous and complete mathematical basis for their application. Therefore, the IOAs need further mathematical analysis in the aspects of distribution density, optimization probability, convergence, complexity, and accuracy of the algorithm. The method proposed in this paper also has this problem. Due to the complexity of the constraints and the variable ranges, the solution space structure is often irregular. Coupled with the influence of other factors, the feasible solution and infeasible solution are often cross-distribution. Some design on operators would lead to too much repetitive and ineffective search. Also, the use of elitism strategy might result in a decrease in the population diversity and ultimately lead to a decline in search performance. To sum up, there are several significant shortcomings. First, the global search and local optimization are not 
easy to balance. Second, it was difficult to maintain population diversity in the search process. Third, there are too many useless operations. Fourth, there are too many ineffective operations. About the difference between the two, see the section 3 of this paper for the details.

Regarding the designing of IOAs, the existing work can be divided into three levels [3]. The primary level is to improve the existing algorithms. For example, according to the characteristics of the problem domain, to extract certain specific rule, and design certain new operator, and so forth. So many original IOAs have appeared some improved versions. The intermediate level is to put forward new search ideas. For example, like GA, the multiple original IOAs proposed by the scholars all have their own unique search patterns. The high level is to break through the traditional idea of intelligent computing. Using a variety of encoding schemes in IOAs is essentially equivalent to mapping the problem to be solved from the current space-time to another. Using the population and individuals to search the optimal solution, is essentially equivalent to using the enumeration method to get the answer. But the use of probability rules makes the emergence of enumeration answers have a certain tendency, that is, the so-called "intelligence" of search behavior. Therefore, it can be said that the GA firstly created the idea of using coded individuals to get the optimal solution via "intelligent enumeration". Comparing with the traditional optimization methods based on strict mathematical logic, this is a kind of brand-new intelligent computation thought. At present, almost all the existing IOAs, including the algorithm framework proposed in this paper, are all failed to escape from this mode of thinking.

\section{Optimization rules implied in the benchmarking philosophy}

As a kind of management approach [2], Benchmarking comes from the field of business management. It is said that it was proposed by Xerox Corporation's predecessor - Haloid Corporation for the first time. However, the author believes that as a management idea, benchmarking came from the traditional culture, especially the traditional management philosophy. Because there are a lot of words in the ancient vocabulary of all ethnic groups, which means that when you see a good man try to emulate him when you see a bad man search you yourself for his fault. The original meaning of benchmark is "A surveyor's mark on a permanent object of predetermined position and elevation used as a reference point", it means a standard by which something can be measured or judged. Benchmarking in the field of enterprise management, the basic idea is to find the learning objects according to the existing achievements and improve the quality of the business via legal learning and effective emulating. In other words, it refers that in order to achieve the purpose of improving its own performance, an enterprise compares against the performance of other companies for the overall operation of the company, or for a business unit, or for a product, or for a department, or for a particular process, and then learns and emulates the best practices among those. The process of benchmarking can be divided into several stages, like setting a benchmark, benchmarking management, resetting the benchmark. In the actual implementation process, the management must be flexible to arrange and adjust due to different situations. Benchmarking, because of its practical effect, since the seventies of last century, it has become a kind of popular management idea and tool in the field of global business management. However, benchmarking is not simply to learn from others, but includes four basic principles, namely, the four core values. That is comprehensive quality, perfect process, reference point and effective learning. Comprehensive quality means to achieve the customer's comprehensive satisfaction. Perfect process means to cover the operation processes of the learning objects besides oneself. The reference point is to set up the common criterion on organizational functions as a basis for comparison. Effective learning means to learn from others and self-learning. According to the connotation of benchmarking, there are several optimization rules implied in the ideas of benchmarking philosophy, which can provide references for the designing of new IOAs.

In business, benchmarking is a process in which a company compares its products and methods with those of the most successful companies in its field, to try to improve its performance. Benchmarking, in short, it is to find the gap with the best case and quickly narrow that gap via 
studying and emulating and eventually go beyond the rival. Therefore, finding the best solution based on benchmarking philosophy is starting from the current existing solution, and through recurring setting benchmark, studying and emulating, resetting benchmark, gradually from the worse option to the bad one, from the sub-optimal option to the optimal one, and ultimately getting the best solution. This is a process of iteration step by step, and this optimization process can be performed in parallel with the help of the modern computing technology and communication technology. It can be seen that from the point of view of optimization, there are a series of optimization rules implied in the benchmarking philosophy.

The principle of setting the benchmark. Setting which one as benchmark depends on the rule of setting the benchmark. As a consequence, according to the characteristics of current problems to be solved, determine the suitable rule of setting benchmark is the first step. In reality, most of the complex problems in the field of science, engineering, and management, ultimately may be transformed into optimization problems. That is, how to construct the model (for example, conceptual model, mathematical model, or another type), and how to solve this model. In the modeling process, firstly, it is necessary to establish the causal relationship between the decision variables and the evaluation objectives. Secondly, it is necessary to define the constraint range of each decision variables. These two parts constitute the model, that is, it is also the evaluation criteria for the alternatives. Take building the mathematical model as an example, whose evaluation function and constraints, constitute the evaluation criteria for decision-making programs. Implementing benchmarking, the first step is to determine the rule of setting the benchmark. That is, setting the proper benchmark according to evaluation criteria for alternative options.

The principle of studying and emulating. After selecting the benchmark, the next step is how to study, emulate and improve. In the real world, all kinds of problems, have the characteristics of their own domain and professionalism. Therefore, in the process of studying and emulating, benchmarking must be carried out based on their own domain features and their own evaluation criteria of the alternative schemes. As outlined in the Brief Analysis and Comment of IOAs in the previous section, the main idea of the IOAs is to use the encoded individuals to get the optimal solution via "intelligent enumeration". Therefore, when an encoding scheme is adopted, then there has its own appropriate principle of studying and emulating. Through studying and emulating, constantly exceeding, constantly updating the alternative programs, making the result continue to be improved and optimized.

The principle of resetting benchmark. After setting the benchmark, followed by studying and emulating, there may be two results. The first is that the desired effect has not been achieved, and another is that the desired effect has been achieved, or even exceeded. In either case, a new benchmark is needed to be set. Many of the decisions in the real world, for a variety of reasons, some did not achieve the positive effects, and some had achieved the desired results. Regardless of the effect of decision-making, the common goal of management decisions in all sectors is ultimate to solve the problem that how to realize sustainable development. Similarly, for the IOA with excellent performance, after the end of an iteration, regardless of the current results, the search results of next iteration are expected to be better, at least not worse than the last. To achieve this goal, it is necessary to re-establish a new benchmark object according to the actual situation of search space.

The principle of teamwork. Once the role model was set up, it became the object of learning and emulating. Especially in the modern enterprise, when benchmarking is put into practice, at least a team, such as a business sector in the company, is the basic unit. This implies achieving common progress through group cooperation in the process of benchmarking. For the IOAs, each individual is relatively simple, and its search ability is relatively limited, however, the group's intelligence is relatively advanced, and the search performance of the group is improved significantly. Therefore, the IOAs, the vast majority of which is based on the group, rather than individual, to carry out searching and optimization.

The principle of diversity. When benchmarking is put into implementation, even if the specific 
industries or areas is explicit, even the specific business or sector is definite, it still requires a certain degree of diversity in alternative schemes and benchmarking objects. Because there are some knowable and controllable factors, especially some unknowable and uncontrollable factors, such as the diversity of decision variables and other reasons. For many problems in the real world, from the standpoint of systematics, maintaining a certain degree of redundancy is conducive to the overall system stability and overall interests. And therefore, it is better that alternative schemes and benchmarking objects have a certain diversity. For the IOAs, if one can maintain the population diversity during the process while running, this just showed its superior performance. On account of their own optimization idea and search strategy, nowadays, most of the IOAs often encountered premature phenomena and faced the challenge of keeping the diversity of population during the search process.

The principle of efficiency. As with other management methods, benchmarking should also pay attention to efficiency issues, that is, input-output ratio. Here assumed that the output effect was fixed, only talk about the cost of inputs. In the real enterprise management, it needs to invest a lot of people, financial, material and other resources in the various stages of benchmarking, and naturally, it is important to try every means to reduce the cost of inputs. For the IOAs, searching for the good candidate solutions, it also needs to invest in costs. For example, the consumption of storage space, run time and other computing resources, of course, the less the better.

\section{Conclusion}

This Paper revealed the optimization rules implied in the benchmarking philosophy, and they will provide good inspirations for the design of intelligent optimization algorithms.

\section{Acknowledgement}

This research is supported by the research fund from "Collaborative innovation center for Transformation and Upgrading of Micro, Small and Medium Enterprises, Zhejiang University of Technology", "Zhejiang Provincial Key Research Base of Philosophy and Social Sciences-Research Centre for Technology Innovation and Enterprise Internationalization", "The National Social Science Fund Project of China(No. 14BJY084)" , "Zhejiang philosophy and social science planning project (Key project , No. 13NDJC004Z)”.

\section{References}

[1] Zhou CH, Xie AS. Dynamic niche-based self-organizing learning algorithm. Journal of Software, 2011,22(8):1738-1748.

[2] What is Benchmarking https://en.wikipedia.org/wiki/Benchmarking.

[3] Xie AS, Yu YD, Huang SM. Optimization algorithm based on benchmarking. Ruan Jian Xue Bao/Journal of Software, 2014, 25(5):953-969 (in Chinese). 